\title{
Recurrent pneumothoraces and facial papules: An insidious pulmonary cystic disease
}

\author{
Dania Nachira $M D^{1}$, Elisa Meacci $M D^{1}$, Leonardo Petracca-Ciavarella MD¹, Pierluigi Novellis MD², \\ Gianluigi Petrone $\mathrm{MD}^{2}$, Stefano Margaritora MD PhD ${ }^{1}$, Filippo Lococo MD², Pierluigi Granone MD PhD ${ }^{1}$
}

\section{CASE PRESENTATION}

A 30-year-old, Caucasian, nonsmoking woman presented with a sixmonth history of bilateral recurrent spontaneous pneumothoraces (PNXs). Previous treatments (bilateral chest drains and iterative chemical pleurodesis) had been attempted; however, only three months after the most recent surgical procedure, the patient presented once again with increasing dyspnea, chest pain, and radiological detection of a right PNX associated with bilateral multiple, thin-walled pulmonary cysts varying in size and shape and predominantly distributed in the lower pulmonary lobes (Figure 1). Physical examination revealed multiple, whitish, smooth, dome-shaped papules on her face (Figure 2) and upper trunk.

Surgery was, thus, indicated and a pulmonary bullectomy combined with a complete right pleural decortication was successfully performed via right thoracotomy.

Intraoperatively, multiple cysts were observed in the lung parenchyma surface (Figure 3A). Histopathological microscopic analysis of the pulmonary cysts (Figure $3 \mathrm{~B}$ ) revealed the presence of so-called 'alveolar cysts' or 'few alveoli within a cyst' (1), with a characteristic pattern marked by the inner surface lined by flattened respiratory epithelium/cuboidal cells (Figure 3C) with signs of chronic inflammation, interstitial bleeding and hyalinization. Ten months later, no radiological signs of recurrent PNX were observed.

Birt-Hogg-Dubé (BHD) syndrome was suspected and confirmed by genetic tests when the patient underwent (at a different centre) rightpartial nephrectomy for renal oncocytoma nine years earlier. The surgical management of recurrent PNX in such cases is an extremely challenging issue: first, because of the failure of standard surgical procedures (as in the present case) and, second, due to the fact that, because bilateral PNXs associated with pulmonary cysts are not extremely rare in young people, BHD syndrome may be confused with other more common entities causing PNX.
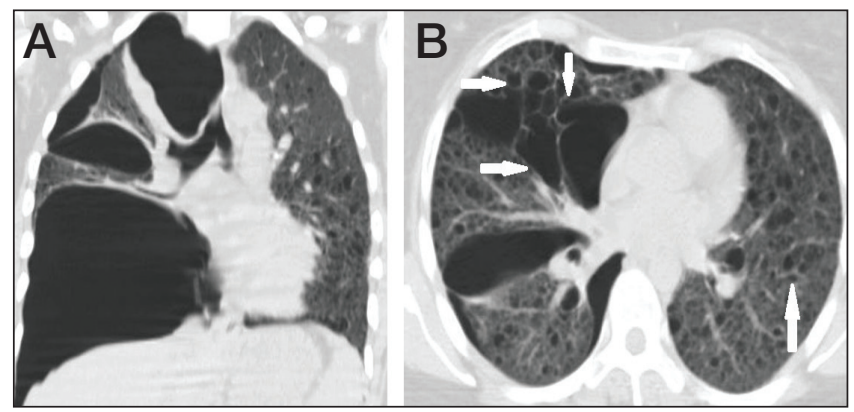

Figure 1) Coronal (A) and axial (B) computed tomography scans of the chest showing a right pneumothorax associated with bilateral multiple, thinwalled pulmonary cysts and lower lung predominant in distribution (arrows)
However, the association of pulmonary cysts (located mostly in the lower pulmonary lobes [2]), the renal tumours and the skin fibrofolliculomas/trichodiscomas represent the typical 'diagnostic triad' (3) of BHD that may help physicians in the diagnostic process.

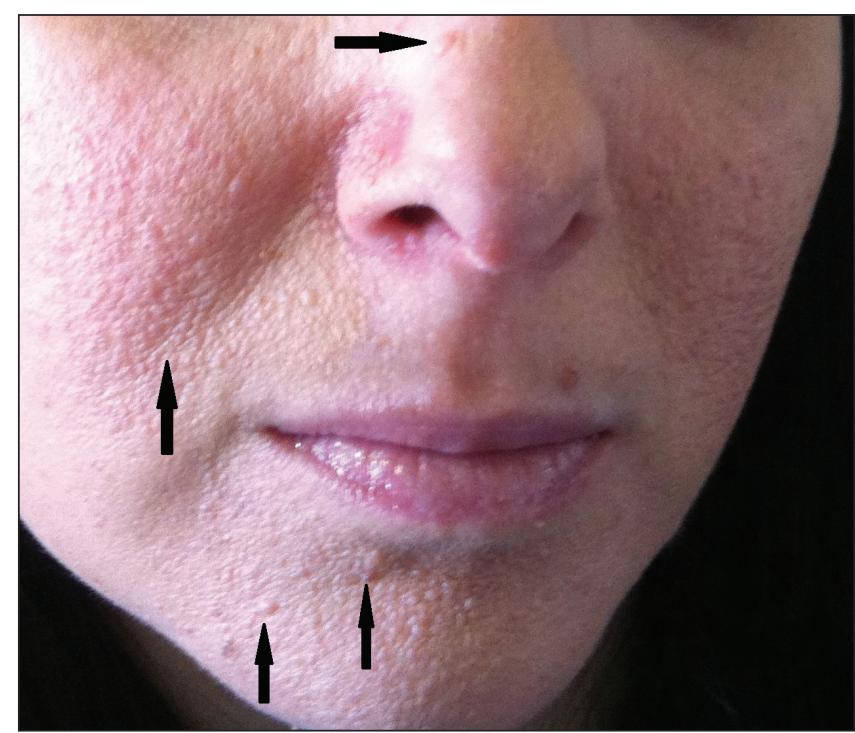

Figure 2) Dome-shaped papules (arrows) on the patient's face

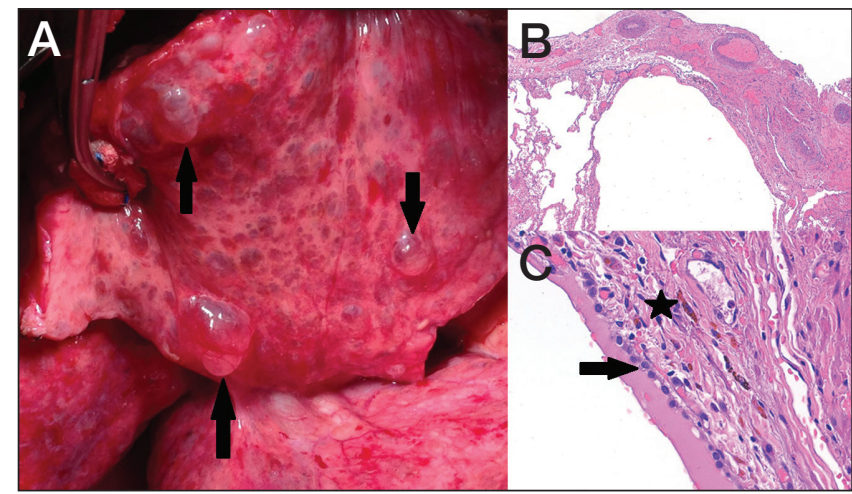

Figure 3) A Intraoperative image of lung parenchyma showing multiple diffusely distributed cysts. B Histopathology of pulmonary cyst specimen, hematoxylin and eosin stain - alveolar cyst, original magnification $\times 50$. C The inner surface of the cyst lined by cuboidal cells (arrow) with signs of chronic inflammation and interstitial bleeding (star). Hematoxylin and eosin stain, original magnification $\times 400$

${ }^{1}$ Department of General Thoracic Surgery; ${ }^{2}$ Department of Pathological Anatomy, Catholic University, Rome; ${ }^{3}$ Unit of Thoracic Surgery, IRCCS-Arcispedale Santa Maria Nuova, Reggio Emilia, Italy

Correspondence: Dr Filippo Lococo, Unit of Thoracic Surgery, IRCCS-Arcispedale Santa Maria Nuova, Reggio Emilia, Italy.

Telephone 00-390-635-6353, e-mail filippo_lococo@yahoo.it 


\section{KEY LEARNING POINTS}

- First described in 1977, BHD syndrome is a rare autosomal dominant multiorgan disorder (prevalence: 0.5 per 100,000 ) caused by mutations in the folliculin gene on chromosome 17p11.2.

- Patients affected by BHD syndrome present with the 'diagnostic triad': skin fibrofolliculomas/trichodiscomas, pulmonary cysts and renal tumours with early onset.

- Lung cysts have been described in $77 \%$ to $89 \%$ of BHD syndrome patients, while the occurrence of PNX could be predicted in approximately $33 \%$ to $38 \%$ of such patients. The right lung is more often affected, although both lungs are involved in approximately $23 \%$ of cases; pulmonary cysts, typically variable in size and shape, are generally located in lower lobes (2).

- The differential diagnosis of BHD syndrome should include other syndromes that may present with PNX and lung cysts such as alpha-1 antitrypsin deficiency, Marfan syndrome, EhlersDanlos syndrome, cystic fibrosis and lymphangio leiomyomatosis.

- In BHD syndrome patients, the treatment of PNX (primary occurrence) consists substantially of standard surgical procedures (chest drain placement); otherwise, in case of recurrent PNX, a pulmonary bullectomy combined with complete pleural decortication should be considered.

\section{REFERENCES}

1. Furuya M, Nakatani Y. Birt-Hogg-Dubé syndrome: Clinicopathological feature of the lung. J Clin Pathol 2013;66:178-86.

2. Agarwal PP, Gross BH, Holloway BJ, Seely J, Stark P, Kazerooni EA. Thoracic CT findings in Birt-Hogg-Dubé syndrome. AJR Am J Roentgenol 2011;196:349-52.

3. Menko FH,van Steensel MA, Giraud S, et al. Birt-Hogg-Dubé syndrome: Diagnosis and management. Lancet Oncol 2009;10:1199-206.

The 'Images in Respiratory Medicine' section of the Canadian Respiratory Journal aims to highlight the importance of visual interpretation, whether physiological, radiological, bronchoscopic, surgical/thorascopic or histological, in the diagnosis of chest diseases. Submissions should exemplify a classic, particularly dramatic or intriguing presentation of a disease while offering an important educational message to the reader (insightful diagnostic pearls or differential diagnosis, etc). This section is not intended to be a vehicle for publication of case reports (see the Clinical-Pathologic Conferences for case-based leaning series). 


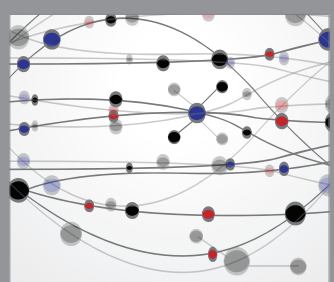

The Scientific World Journal
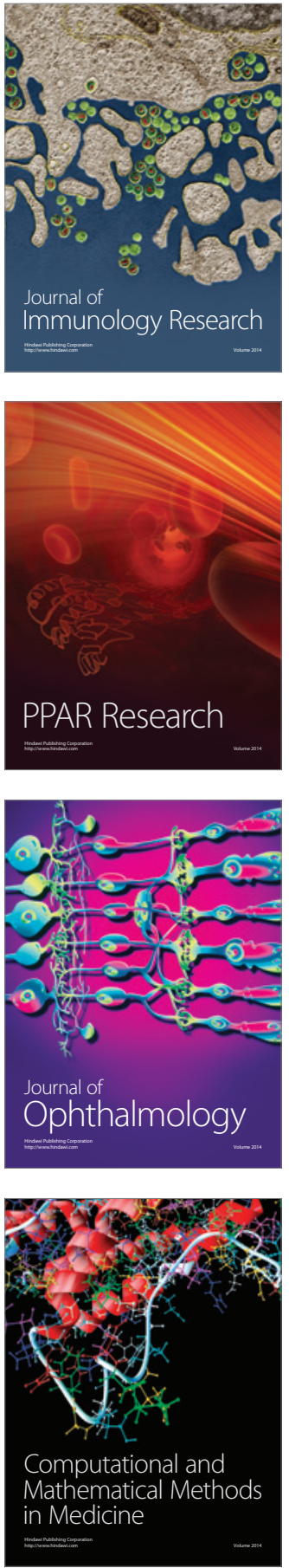

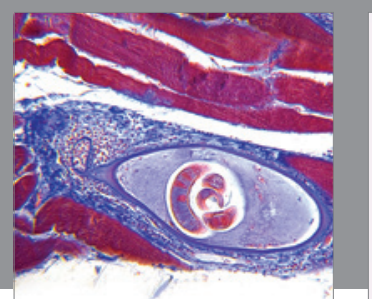

Gastroenterology Research and Practice

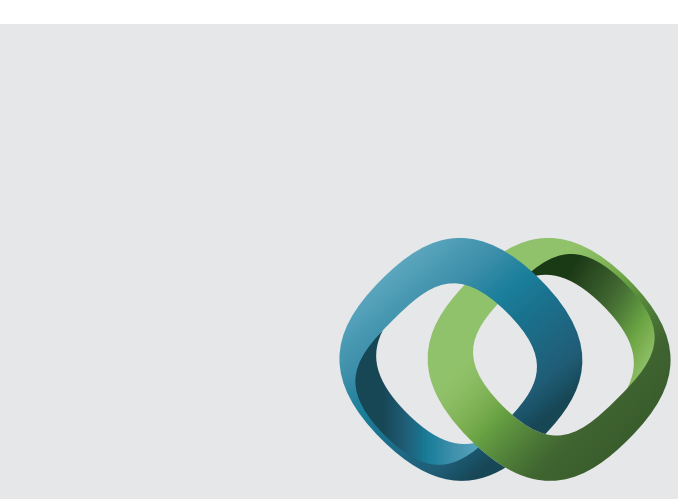

\section{Hindawi}

Submit your manuscripts at

http://www.hindawi.com
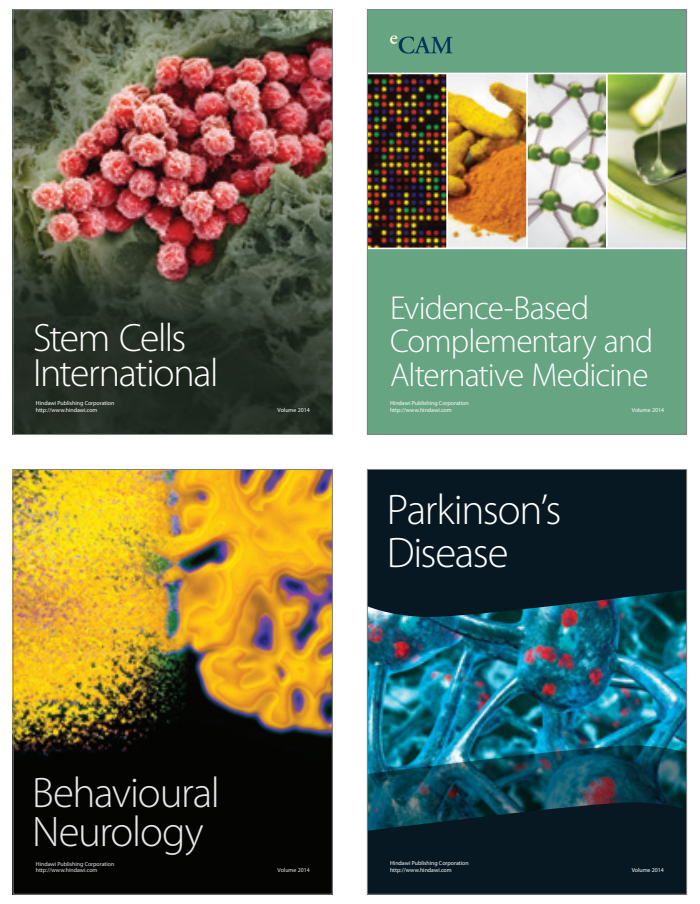
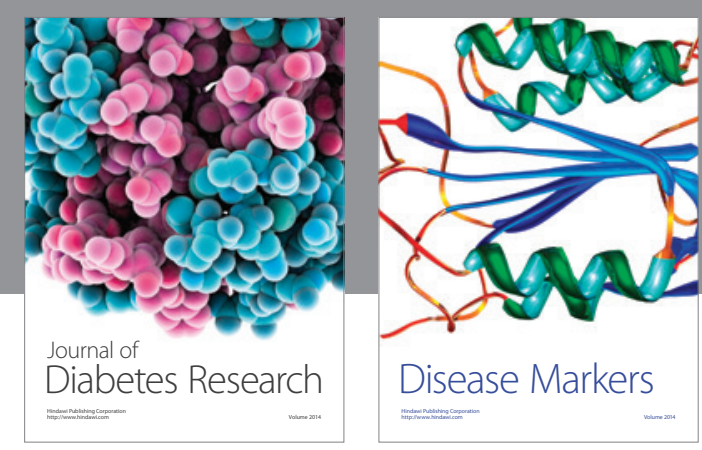

Disease Markers
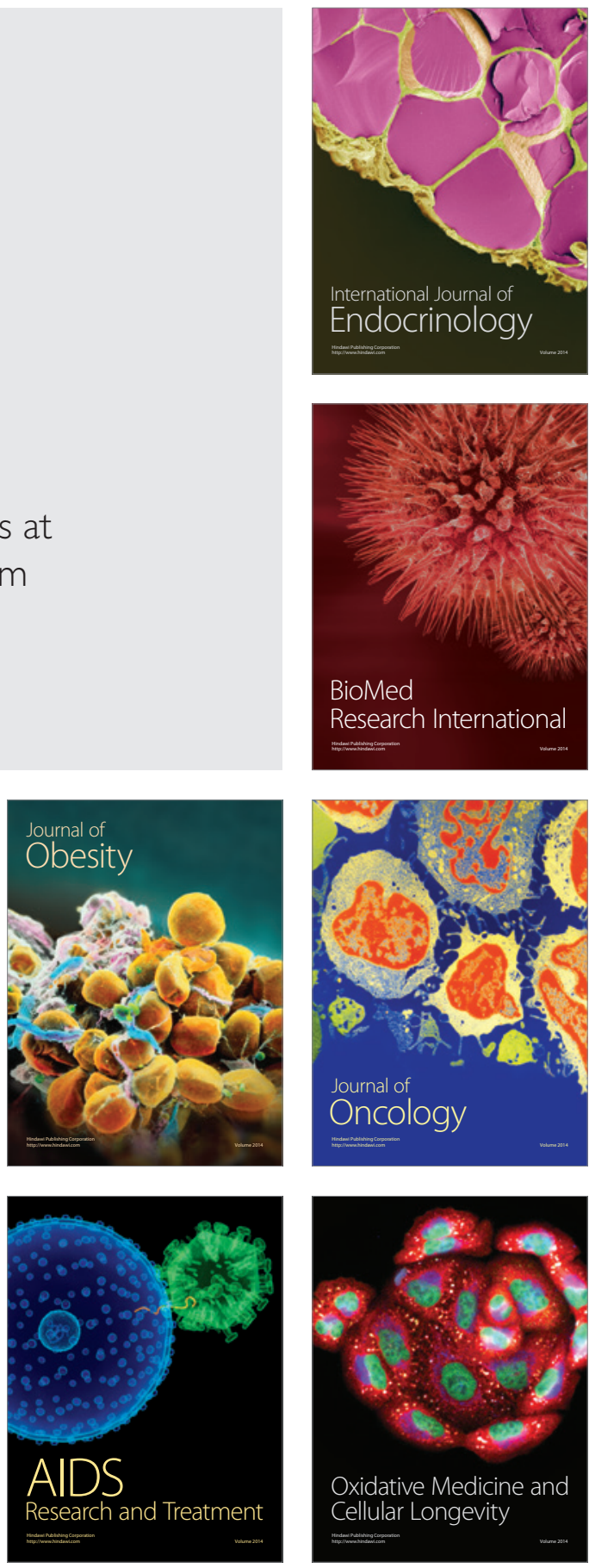